\title{
MULTI-MATCHER ON-LINE SIGNATURE VERIFICATION SYSTEM IN DWT DOMAIN
}

\author{
Isao Nakanishi \\ Tottori University \\ Faculty of Regional Sciences \\ 4-101 Koyama-minami, Tottori, 680-8551 Japan
}

\author{
Hiroyuki Sakamoto, Yoshio Itoh, Yutaka Fukui
}

\author{
Tottori University \\ Faculty of Engineering \\ 4-101 Koyama-minami, Tottori, 680-8552 Japan
}

\begin{abstract}
This paper presents a multi-matcher on-line signature verification system which fuses the verification scores in pen-position parameter and pen-movement angle parameter at decision level. Features of pen-position and pen-movement angle are extracted by the sub-band decomposition using the Discrete Wavelet Transform (DWT). In the pen-position, high frequency sub-band signals are considered as individual features to enhance the difference between a genuine signature and its forgery. On the other hand, low frequency sub-band signals are utilized as the features for suppressing the intra-class variation in the pen-movement angle. Verification is achieved by the adaptive signal processing using the extracted features. Verification scores in the pen-position and the pen-movement angle are integrated by using a weighted sum rule to make total decision. Experimental results show that fusion of pen-position and pen-movement angle can improve verification performance.
\end{abstract}

\section{INTRODUCTION}

Single biometric systems may not be always applicable because of unacceptable performance and inability to operate on a large user population. Multiple biometric systems can overcome these limitations [1]-[6]. Five scenarios of the multiple biometric system have been proposed in [3], that is, multi-sensor system, multimodal system, multi-unit system, multi-impression system, and multi-matcher system. Among of them, the multi-matcher system which uses multiple representation and matching algorithm for the same input biometric signal is the most cost-effective way to improve the performance of the biometric system [3].

We have proposed the on-line signature verification system in the Discrete Wavelet Transform (DWT) domain [7]. This system utilized only pen-position parameter since it was detectable even in portable devices such as the Personal Digital Assistants (PDA). A time-varying signal of pen-position parameter was decomposed into sub-band signals by using the DWT. Verification was achieved by using the adaptive signal processing in each sub-band. Total decision for verification was done by combining such verification results. Verification rate of about $95 \%$ was obtained, which was improved by about $10 \%$ comparing with a time-domain verification system.

In this paper, we introduce multi-matcher scheme into our online signature verification system. In addition to pen-position parameter, pen-movement angle parameter is processed by the DWT and the adaptive signal processing to obtain verification results in sub-bands. The pen-movement angle parameter is derived from the pen-position parameter; therefore, the proposed system requires no additional sensor. While high frequency sub-band signals are treated as individual features in the pen-position to enhance the difference between a genuine signature and its forgery, low frequency sub-band signals are utilized as the features in the pen-movement angle for suppressing the intra-class variations in signatures of one individual. Verification results of both the pen-position and the pen-movement angle are integrated at total decision level.

\section{ON-LINE SIGNATURE VERIFICATION IN DWT DOMAIN}

The on-line signature is digitized with the electronic pen-tablet. Especially, we utilize only pen-position parameter since it is provided even in such as the PDA for handwriting or pointing. Actually, the pen-position parameter consists of discrete time-varying signals of $\mathrm{x}$ and y coordinates, which are $x^{*}\left(n^{\prime}\right)$ and $y^{*}\left(n^{\prime}\right)$, respectively. $n^{\prime}\left(=0,1, \cdots, N_{\max }-1\right)$ is a sampled time index. $N_{\max }$ is the total number of sampled data. As the one-line signature is a dynamic biometrics, each writing time is different from the others. This results in the different number of sampled data even in genuine signatures. Moreover, different writing place and different size of signature cause variations in pen-position parameter. To reduce such variations, pen-position data are normalized in general. The normalized pen-position parameter is defined as

$$
x(n)=\frac{x^{*}(n)-x_{\min }}{x_{\max }-x_{\min }} . \quad y(n)=\frac{y^{*}(n)-y_{\min }}{y_{\max }-y_{\min }} \cdot \alpha_{y}
$$

where $n(=0 \sim 1)$ is a normalized sampled time index given by $n=n^{\prime} /\left(N_{\max }-1\right) . \quad x_{\max }$ and $y_{\max }$ are maximum and minimum values of $x^{*}(n)$ and $y^{*}(n)$, respectively. $\alpha_{x}$ and $\alpha_{y}$ are scaling factors for avoiding underflow calculation in sub-band decomposition described later.

However, such normalization makes the difference between a genuine signature and its forgery unclear [7]. In addition, the on-line signature is relatively easy to forge if the written signature is known. Easiness of imitating pen-position data decreases the difference between the genuine signature and the forgery further. Therefore, it is not easy to distinguish between the genuine signature and the forgery by using the time-varying signal of a pen-position parameter [7].

In order to enhance the difference between a genuine signature and its forgery, we have proposed to verify the on-line signature in DWT domain [7]. In the following, $x(n)$ and $y(n)$ are represented as $v(n)$ for convenience. The DWT of the normalized pen-position $v(n)$ is defined as

$$
u_{k}(m)=\sum_{n} v(n) \overline{\Psi_{k, m}(n)}
$$




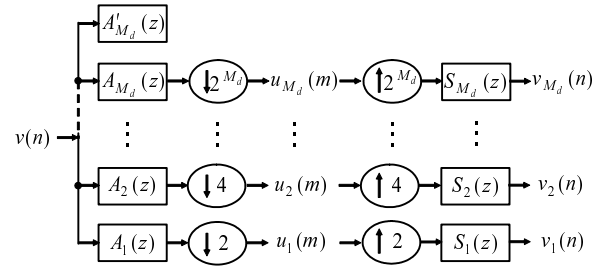

Fig. 1. Parallel structure of sub-band decomposition by DWT.

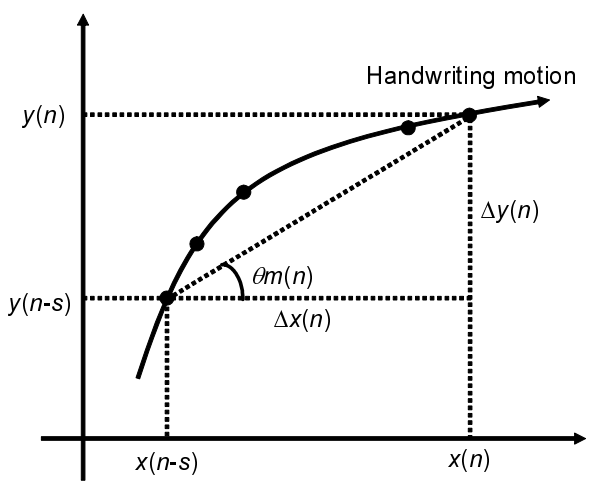

Fig. 2. Definition of pen-movement angle $\theta(n)$.

where $\Psi_{k, m}(n)$ is a wavelet function and ${ }^{-}$denotes conjugate. $k$ is a frequency (level) index.

Moreover, it is well known that the DWT corresponds to the octave-band filter bank. Fig.1 shows a parallel structure of the subband decomposition. $M_{d}$ is decomposition level. The synthesized signal $v_{k}(n)$ in each sub-band is called Detail. The Detail is the signal in high frequency band and so it contains differences between signals. Therefore, we consider the Detail as an enhanced individual feature in pen-position.

Results by sub-band decomposition of pen-position parameter are omitted for duplication. The difference between a genuine signature and its forgery can be enhanced in the DWT domain. Please refer to [7] in detail.

\section{FUSION OF PEN-POSITION AND PEN-MOVEMENT ANGLE}

For improving verification performance, the multi-matcher system is the most cost-effective [3]. In this paper, we propose a new pen-movement angle parameter and to fuse it with a conventional pen-position one.

\subsection{Pen-movement Angle}

We define the pen-movement angle parameter $\theta(n)$ in Fig.2 and Eq.(3).

$$
\begin{gathered}
\theta(n)= \begin{cases}\tan ^{-1} \frac{\Delta y(n)}{\Delta x(n)}, & \Delta x(n)>0 \\
\tan ^{-1} \operatorname{sgn}(\Delta y(n)) \cdot \frac{\pi}{2}, & \Delta x(n)=0 \\
\tan ^{-1} \frac{\Delta y(n)}{\Delta x(n)}+\pi, & \Delta x(n)<0, \Delta y(n) \geq 0 \\
\tan ^{-1} \frac{\Delta y(n)}{\Delta x(n)}-\pi, & \Delta x(n)<0, \Delta y(n)<0\end{cases} \\
\Delta x(n)=x(n)-x(n-s), \Delta y(n)=y(n)-y(n-s)
\end{gathered}
$$

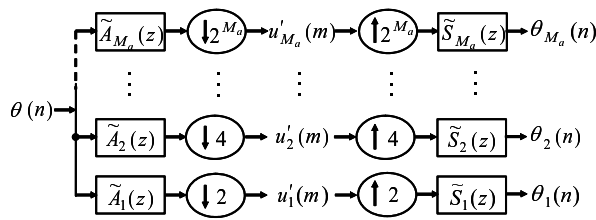

Fig. 3. Sub-band filter bank for extracting Approximations.

where $s$ presents amount of time shift.

The pen-movement angle parameter is derived from the penposition one; therefore, the proposed multi-matcher system requires no additional sensor. Furthermore, the pen-movement angle parameter essentially has two-dimensional characteristics. As a result, it brings more obvious individual feature of the on-line signature than the pen-position parameter which is actually in onedimensional.

\subsection{Suppressing Intra-class Variations}

It is also confirmed that the proposed pen-movement angle parameter have large intra-class variation in signatures of one individual. For utilizing the pen-movement angle parameter in verification, some reduction method of the intra-class variation is required.

In order to suppress such intra-class variations, we extract an Approximation as an enhanced similarity of the pen-movement angle parameter. The Approximation is the low frequency band signal in sub-band decomposition by the DWT; therefore, it contains similarity between signals.

Fig.3 shows the sub-band filter bank for extracting the Approximations. $\tilde{A}_{k}(z)$ and $\tilde{S}_{k}(z)$ where $k=1, \cdots, M_{a}$ are the synthesis filter and the analysis filter, respectively. $M_{a}$ is decomposition level in the pen-movement angle.

Examples of the Approximation are shown in Fig.4. Fig.4(a) indicates that the similarity of two genuine signatures is extracted in Approximations. On the other hand, Fig.4(b) shows that the difference between a genuine signature and its forgery is kept even in Approximations. These comparisons suggest that the verification using the Approximation can suppress the intra-class variation keeping the difference of the genuine signature and its forgery.

\subsection{Multi-Matcher Verification System}

By using pen-position and pen-movement angle parameters, we propose a new multi-matcher on-line signature verification system. Fig. 5 shows a system overview. Pen-position, actually $\mathrm{x}$ and $\mathrm{y}$ coordinates and pen-movement angle are separately processed in verification block. The verification block is common to processing of pen-position and pen-movement angle. Fig. 6 describes the verification block, where pen-information, that is, pen-position and pen-movement angle parameters are decomposed into Details or Approximations and then they are verified with templates using the adaptive signal processing at each level.

Before verification, templates must be enrolled in order to be compared with input signatures. As the template, $T$ genuine signatures which have equal number of strokes are prepared and then their pen-position and pen-movement angle parameters are decomposed into sub-band signals by the DWT separately. Decomposition level is decided after examinations of those genuine signatures. Extracted $T$ Details for the pen-position and $T$ Approxima- 


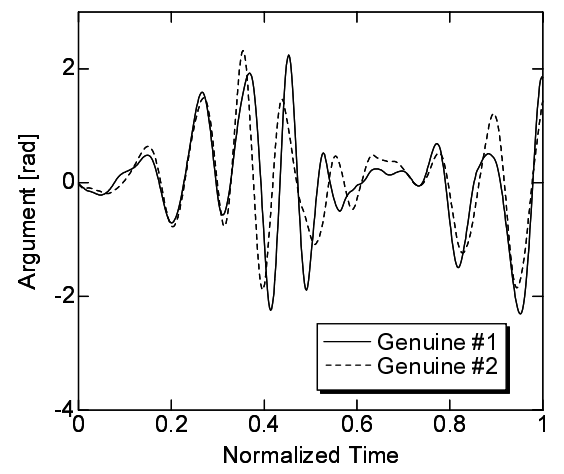

(a) Comparison between genuine signatures

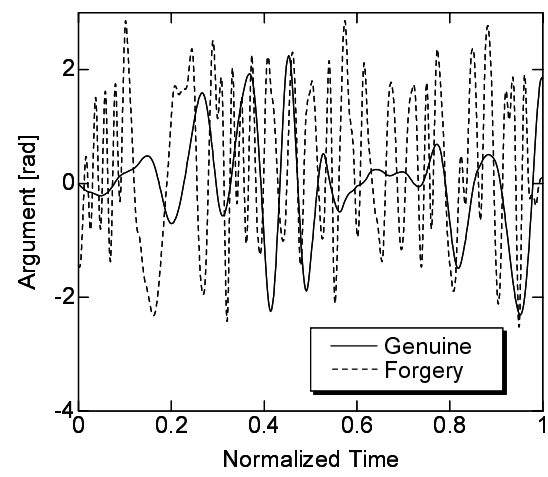

(b) Comparison between a genuine signature and Its forgery

Fig. 4. Examples of Approximation in pen-movement angle.

tions for the pen-movement angle are averaged at the same level each other.

By the way, if the number of strokes in an input signature is different from that in a template, it is natural to consider the input signature as a forgery. However, not all genuine signatures have the same number of strokes. We adopt the dynamic programming (DP) matching to identify the number of strokes in an input signature with that in a template. The procedure of the stroke matching is omitted for lack of space. It is described detailedly in [7].

\subsection{Verification Using Adaptive Signal Processing}

After enrollment phase, verification phase is executed. The verification is achieved by using the adaptive signal processing. The purpose of the adaptive signal processing is to reduce the error between the input signal and the desired signal sample by sample. When an input signal is of a genuine signature, the error between the input and its template becomes small; therefore, adaptive weights are expected to converge close on 1 . Inversely, if the input signature is a forgery, adaptive weights converge far from 1. In this way, the verification can be achieved by examining whether converged value is nearly 1 or not [7].

As the adaptive algorithm, we use a new kind of steepest descent algorithm defined as follows.

$$
\begin{gathered}
w_{k}(n+1)=w_{k}(n)+\mu E\left[e_{k}(n) \rho_{k}(n)\right] \\
e_{k}(n)=t_{k}(n)-w_{k}(n) \rho_{k}(n)
\end{gathered}
$$

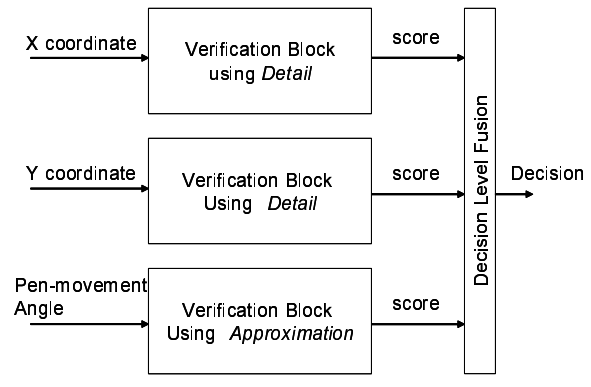

Fig. 5. Proposed multi-matcher verification system.

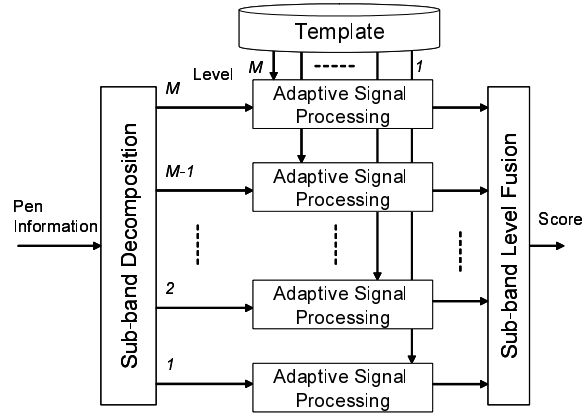

Fig. 6. Verification block

$$
\begin{gathered}
E\left[e_{k}(n) \rho_{k}(n)\right]=\frac{1}{N_{t m p}} \sum_{l=0}^{N_{t m p}-1} e_{k}(r-l) \rho_{k}(r-l) \\
\mu=\mu_{0} /\left\{E\left[\left|\rho_{k}(n)\right|\right]\right\}^{2} \\
E\left[\left|\rho_{k}(n)\right|\right]=\frac{1}{I} \sum_{l=0}^{I-1} \rho_{k}(n-l)
\end{gathered}
$$

where $I$ is the number of sampled data in an input Detail or Approximation. $N_{t m p}$ is the number of sampled data in a template. $\mu$ is a step size parameter which controls the convergence in the adaptive algorithm. The step size parameter is normalized by input power as shown in Eqs.(7) and (8), so that convergence is always guaranteed. $\mu_{0}$ is a positive constant.

The verification is done in all sub-bands in parallel. After enough iterations for convergence, $w_{k}(n)$ is averaged in past $N_{t m p}$ samples and then we obtain the converged value $w_{k}$. A verification score is obtained by fusing several sub-band level results. In this paper, such sub-band level fusion is achieved by averaging the converged values.

$$
\begin{aligned}
& \text { Score }^{x}=\frac{1}{L_{d}} \sum_{k=M_{d}-L_{d}+1}^{M_{d}} w_{k}^{x} \\
& \text { Score }^{y}=\frac{1}{L_{d}} \sum_{k=M_{d}-L_{d}+1}^{M_{d}} w_{k}^{y} \\
& \text { Score }^{\theta}=\frac{1}{L_{a}} \sum_{k=M_{a}-L_{a}+1}^{M_{a}} w_{k}^{\theta}
\end{aligned}
$$

where $w_{k}^{x}, w_{k}^{y}$ and $w_{k}^{\theta}$ respectively denote the converged values of $\mathrm{x}, \mathrm{y}$ and pen-movement angle at level $k . L_{d}$ is the used level 
number in decision fusion of pen-position and $L_{a}$ is that of penmovement angle.

\subsection{Decision Fusion}

As shown in Fig.5, verification results are fused and then we obtain a total decision. There have been proposed many fusion methods such as the sum rule, the minimum score, the maximum score, the neural networks and so on [1]-[6]. In this paper, we employ the sum rule in which scores by pen-position and pen-movement angle are weighted and then summed. The total decision for verification is defined as

$$
\begin{array}{r}
\mathrm{TC}=c_{x} \cdot \text { Score }^{x}+c_{y} \cdot \text { Score }^{y}+c_{\theta} \cdot \text { Score }^{\theta} \\
c_{x}+c_{y}+c_{\theta}=1, \quad c_{x}>0, c_{y}>0, c_{\theta}>0
\end{array}
$$

$c_{x}$ and $c_{y}$ denote the weighting factors for $\mathrm{x}$ and $\mathrm{y}$ coordinates, respectively and $c_{\theta}$ is that for pen-movement angle. These are determined in preliminary examinations. When the TC is greater than a threshold, an input signature is decided to be genuine.

\section{EXPERIMENTAL RESULTS}

Four subjects were requested to sign their own signatures and then we obtained 118 genuine signatures. Five genuine signatures for each subject were used to make a template and the remaining 98 genuine signatures were used for verification. Five subjects were required to counterfeit the genuine signature 10 times each, so that 200 forgeries were prepared in total. Daubechies8 was used as a wavelet function since it was confirmed to be the best by trial and error in preliminary examinations. Other conditions of simulation are summarized as follows.

- Scaling parameter: $\alpha_{x}=\alpha_{y}=100$

- Number of signatures for making a template: $T=5$

- Upper limit decomposition level: $M_{d}^{\max }=8$

- Decomposition level in pen-movement angle: $M_{a}=4$

- Number of processed level: $L_{d}=4, L_{a}=2$

- Step size constant: $\mu_{0}=0.0001$

- Number of iterations: $10^{5}$

- Time shit in pen-movement angle: $s=8$

- Weighting factor: $c_{x}=0.4, c_{y}=0.2, c_{\theta}=0.4$

Fig. 7 shows the variation of False Rejection Rate (FRR) and False Acceptance Rate (FAR) with total decision threshold. In general, verification performance is estimated by Equal Error Rate (EER) where the FRR is equal to the FAR. The EER was about $3.3 \%$ when the threshold value was about 0.30 . Verification rate was about $96.7 \%$. Comparing with our conventional on-line signature verification method in which only pen-position is processed [7], the verifiaction performance was improved by about $1.7 \%$.

\section{CONCLUSION}

We have presented a multi-matcher on-line signature verification system. Both pen-position and pen-movement angle parameters were decomposed into sub-band signals by the DWT. Moreover, high frequency sub-band signals called Detail were extracted as individual features in pen-position to enhance the difference between

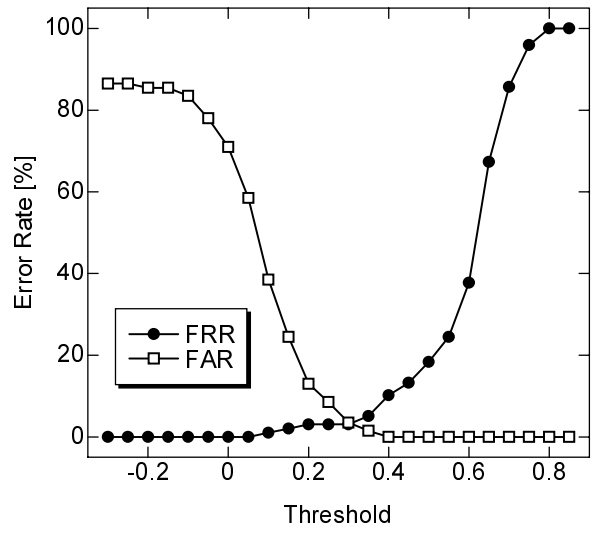

Fig. 7. Variation of FAR and FRR with decision threshold.

a genuine signature and its forgery while low frequency sub-band signals called Approximation were used for pen-movement angle to suppress the intra-class variation of signatures in one individual. Verification results of pen-position and pen-movement angle were combined at decision level. We demonstrated that the proposed multi-matcher scheme improved the performance of the on-line signature verification system in the DWT domain.

Though the proposed system requires no additional sensor, computational complexity becomes twice as much as that of the conventional system because the computational complexity for verification using the pen-movement angle parameter is equivalent to that using the conventional pen-position parameter.

To reduce the computational complexity must be studied in future. Also, verification experiments using large number of signatures is a problem.

\section{REFERENCES}

[1] J. Kittler, J. Matas, K, Jonsson, and M.U. Ramos Sánchez, "Combining evidence in personal identity verification systems," Pattern Recognition Letters, vol.18, pp.845-852, 1997.

[2] L. Hong and A. K. Jain, "Multimodal Biometrics in BIOMETRICS Personal Identification in Networked Society", ed. A. Jain, R. Boll, and S. Pankanti, pp.327-344, Kluwer Academic Publishers, Massachusetts, 1999

[3] S. Prabhakar, A. K. Jain, "Decision-level Fusion in Fingerprint Verification," Pattern Recognition, vol.35, pp.861-874, 2002.

[4] A. Ross and A. Jain, "Information fusion in biometrics," Pattern Recognition Letters, vol.24, pp.2115-2125, 2003.

[5] M. Indovina, U. Uludag, R. Snelick, A. Mink, and A. Jain, "Multimodal Biometric Authentication Methods: A COTS Approach," Proc. of Workshop on Multimodal User Authentication, Santa Barbara, USA, pp.99-106, Dec. 2003.

[6] A. Kumar and D. Zhang, "Integrating Palmprint with Face for User Authentication," Proc. of Workshop on Multimodal User Authentication, Santa Barbara, USA, pp.107-112, Dec. 2003.

[7] I. Nakanishi, N. Nishiguchi, Y. Itoh, and Y. Fukui, ”Online Signature Verification Method Based on Discrete Wavelet Transform and Adaptive Signal Processing," Proc. of Workshop on Multimodal User Authentication, Santa Barbara, USA, pp.207-214, Dec. 2003. 